\title{
Stochastic Path Model of Polaroid Polarizer for Bell's Theorem and Triphoton Experiments
}

\author{
Paul J. Werbos* \\ ECCS Division, National Science Foundation
}

\begin{abstract}
Depending on the outcome of the triphoton experiment now underway, it is possible that the new local realistic Markov Random Field (MRF) models will be the only models now available to correctly predict both that experiment and Bell's Theorem experiments. The MRF models represent the experiments as graphs of discrete events over space-time. This paper extends the MRF approach to continuous time, by defining a new class of realistic model, the stochastic path model, and showing how it can be applied to ideal polaroid type polarizers in such experiments. The final section discusses possibilities for future research, ranging from uses in other experiments or novel quantum communication systems, to extensions involving stochastic paths in the space of functions over continuous space. As part of this, it derives a new Boltzmann-like density operator over Fock space, which predicts the emergent statistical equilibria of nonlinear Hamiltonian field theories, based on our previous work extending the Glauber-Sudarshan P mapping from the case of classical systems described by a complex state variable $\alpha$ to the case of classical continuous fields.
\end{abstract}

\section{Introduction}

The original Bell's Theorem experiments [1] were correctly predicted by use of an early form of quantum mechanics, which modeled the polarizers in the experiment as lumped circuit elements performing a "collapse of the wave function." In two previous papers [2,3], we reviewed that work, provided a more modern quantum mechanical description of the collapse of the wave function without metaphysical observers, and derived the predictions for a new experiment we proposed, the triphoton experiment now underway at Maryland $[4,5]$. We also provided three alternative local realistic Markov Random Field (MRF) models of the Bell's Theorem experiments - MRF1, MRF2 and mRF3 which give predictions for the triphoton experiment quite different from those given by assuming collapse of the wave function. In particular, the MRF models predict [2] a three-photon counting rate difficult or impossible to reconcile with traditional intuitive ideas about time, either from classical physics or from traditional time-asymmetric versions of quantum mechanics:

$$
\mathrm{R}_{3} / \mathrm{R}_{0}=\mathrm{k} \cos ^{2}\left(\theta_{\mathrm{c}}-\theta_{\mathrm{a}}-\theta_{\mathrm{b}}\right)
$$

This paper addresses the question: if equation 1 is vindicated by experiment, and if the collapse of the wave function itself collapses, what can we replace it with, to build a bridge between the underling physics of these experiments and their observed outcomes? In an earlier paper [6], we discussed the issue of how to inject and exploit time-symmetry either into realistic models in $3+1$ dimensions, or into many-worlds versions of quantum field theory $[7,8]$, either one of which could be modified to accommodate the new experimental results. This paper will mainly focus on the first approach, the realistic approach, because it is easier to implement and to connect to these experiments; however, section 4 will discuss more general issues, including some which are common to both approaches.

For experiments using crystal polarizers like calcite, the MRF3 model already gives a reasonable first-order picture of how the physics of a polarizer can be reconciled with the outcome of Bell's Theorem experiments. It provides a realistic explanation of what actually happens in those experiments. (Here, we use the word "realistic" in the technical sense $[2,3,9]$, not as synonym for "plausible," though we do also believe it is plausible as a first-order picture.) However, what about other experiments in quantum optics, where a graph of discrete events is not powerful enough for accurate modeling? What of systems like polaroid polarizers, where a continuous time model is needed even for a serious first-order understanding of the physics?

\footnotetext{
${ }^{11}$ The views herein represent no one's official views, but the paper was written on US government time
} 
Section 2 of this paper will present and explain a new general modeling framework, the stochatsic path approach, which generalizes the MRF modeling of $[2,3]$ to the case of continuous time. Section 3 will present a stochastic path model of the polaroid polarizer, and show how it matches the successful predictions of MRF1 for Bell's Theorem experiments (with brief comment on where equation comes from). Further possibilities for the use of the stochastic path method are dicussed in the begtining of section 4 .

The first stochastic path approach, in sections 2 and 3, uses a finite number of variables to describe the real state of a system at any time t, as do many other important methods: ordinary or stochastic differential equations $[10,11,12]$; the "first quantization" before the development of quantum field theory; the original Glauber-Sudarshan "P" mapping of quantum optics $[13,14,15]$; and the Feynman path approach before the generalization to functional

integrals. Section 4 will discuss an extension of the stochastic path approach to the case of fields over continuous space, making use of my extension [15] of the Glauber-Sudarshan mapping to classical Hamilton field theories. It discusses how to extend the stochastic path approach to predictions of open systems experiments using these methods, either for ensembles of classical fields or for density matrices in the many-worlds version of quantum mechanics. It derives a new Boltzmann formula for the equilibrium statistics of a wide class of such field theories, similar but not identical to the usual grand canonical density matrix of quantum statistical thermodynamics.

\subsection{Introduction}

\section{Stochastic Path Models}

Like the Feynman path method for formulating quantum models, the stochastic path method can be approached from several different directions. Formally, a stochastic path model is any well-defined stochastic model for a system defined by $\mathrm{X}(\mathrm{t})$, where $\mathrm{X}$ is a discrete set of state variables across time t, obeying a Markov constraint on correlations across time, and obeying certain constraints on time symmetry [6]. The state variables may be continuous or discrete, and may go through times of simply being undefined (like the polarization of a photon which was absorbed at an earlier time).

In this section, we will explain the idea inductively, by first discussing the general principles embedded in our previous MRF models [2,3], and showing how to take the continuous limit. We will also cite previous lengthy discussions of the concepts of causality, locality and realism which are fundamental to all discussions of Bell's Theorems and models capable of correctly predicting Bell's Theorem experiments.

Intuitively, the original Feynman path method was also a method for modeling and predicting possible patterns or paths $\{\mathrm{X}(\mathrm{t})\}$ across time, defined by the probability amplitude formula underlying it all:

$$
\psi(\{X(t)\})=k e^{i \hbar \int L(X(t)) d t}
$$

The stochastic path method can be equivalently defined as the same idea, but with:

$$
\operatorname{Pr}(\{X(t)\})=\frac{1}{Z} e^{-\int L(X(t)) d t}
$$

using real probabilities, normalized by a partition function $\mathrm{Z}$ such that the probabilities add up to one. Equation 3 empodies the idea of stochastic realism.

Neither of these equations, 2 or 3 , represents a march of probabilities forward in time from time $-\infty$ to $+\infty$. Both allocate probabilities according to properties of an entire possible course of events over past and future. Neither leads to predictions which are consistent with more classical ideas of how time works, or with the classical statistical assumptions used to define "causality" in Bell's Theorem proofs $[1,2,3]$. Nevertheless, both systems imply a kind of mathematical causality which can we use in making predictions of experiments.

The MRF models which we have described in the past can be formulated as the special case of equation 3, in which we only need to consider a finite set of event times, and not a continuum. The remarkable finding here is that models which satisfy this kind of stochastic realism have not only been able to predict the outcome of Bell's Theorem; they have also been able to predict nonclassical counterintuitive outcomes for a new triphoton experiment[2] which nothing else has been able to do. 


\subsection{Essential Properties of Physically Plausible MRF Models}

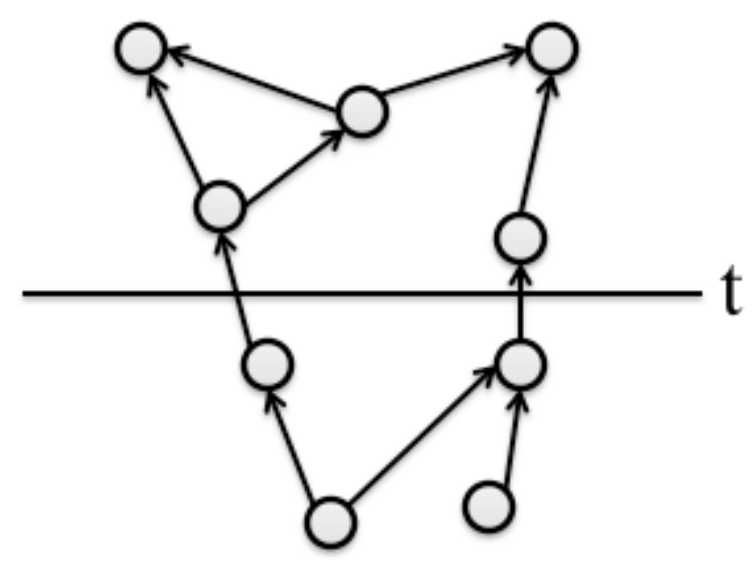

Figure 1. A graph of events across space-time

Figure 1 illustrates the general concept of a graph of connected events across space-time. See [2] and [3] for concrete examples, where the events may be the emission of two entangled photons, entry or exit of a photon from a polarizer, or absorption or detection or rotation of a photon.

The nodes representing events are numbered from 1 to $\mathrm{N}$. The overall path or outcome is defined by a collection of variables, $\mathrm{X}$, which are not indexed by time in the MRF formalism itself, though they may include variables like $\theta_{-}^{\mathrm{L}}$, the linear polarization of the photon on the left channel at the time before it reaches the left polarizer. The probability of any possible outcome $\mathrm{X}$ is defined by the model:

$$
\operatorname{Pr}(\mathrm{X})=(1 / \mathrm{Z}) \mathrm{p}_{1} *(\mathrm{X}) \mathrm{p}_{2} *(\mathrm{X}) \ldots \mathrm{p}_{\mathrm{N}} *(\mathrm{X})=\operatorname{Pr}^{*}(\mathrm{X}) / \mathrm{Z}
$$

where the partition factor $\mathrm{Z}$ is just the sum of $\operatorname{Pr}^{*}(\mathrm{X})$ over all possible outcomes $\mathrm{X}$, and is used to make sure that the final probabilities $\operatorname{Pr}(\mathrm{X})$ add up to 1 . MRF models based on equation 4 have been widely used in many fields, such as computational intelligence. The node functions $\mathrm{p}_{\mathrm{k}}{ }^{*}(\mathrm{X})$ also have many names. Here, I will refer to these functions as endogenous time-symmetric probabilities (ETSP), because of the physical requirement that they should be timesymmetric for all nodes except those which represent an injection of positive-time free energy into the system from the outside [6]. The requirement for time symmetry violates classical intuition, as expressed by Einstein, Podolosky and Rosen, but it is impossible for a local realistic model to correctly predict Bell's Theorem experiments without violating that intuition. In [2] and [3], we were not as strict about the symmetry constraint as we will be here, because it would have required adding terms which would not have affected the predictions in any case.

To map equation 4 into equation 3, replace the integration over time in equation 3 with a sum over the $\mathrm{N}$ discrete times here, and define $\mathrm{L}\left(\mathrm{t}_{\mathrm{k}, \mathrm{X}}\right)$ as $\log \mathrm{p}_{\mathrm{k}}{ }^{*}(\mathrm{X})$.

Nevertheless, for the benefit of those who like to think in time-forwards terms, and for use in calculations, equation 4 can also be represented equivalently as the outcome of a time-forward recurrence relation:

$$
\mathrm{P}_{\mathrm{k}+1}^{+}(\mathrm{X})=\left(1 / \mathrm{Z}_{\mathrm{k}+1}\right) \mathrm{P}_{\mathrm{k}}^{+}(\mathrm{X}) \mathrm{p}_{\mathrm{k}}{ }^{*}(\mathrm{X})
$$

where the dynamic partition factor $\mathrm{Z}_{\mathrm{k}+1}$ normalizes the probabilities $\mathrm{P}_{\mathrm{k}+1}{ }^{+}(\mathrm{X})$ to add up to one. If we use the ETSP models $\mathrm{p}_{1}{ }^{*}, . . \mathrm{p}_{\mathrm{N}} *$ from the MRF1 or MRF3 models, does equation 5 then give us a classically "causal" model of the progression of the Bell Experiment from starting time to ending time, disproving the theorem that a successful local, realistic, "causal" model is impossible? No, because Bayesian convolution as in equation 5 does not obey the definition of "locality" used in those theorems. Equation 5 allows for the possibility that evidence in one place changes our estimate of the probability of a variable active in another place (even when the graph in figure 1 involves local connections or edges); thus it is "nonlocal" in that technical sense. The partition factor in equation 5 is the main vehicle for introducing this kind of "nonlocality"; it may seem small and simple, but it has a huge impact on howthese kinds of dynamical systems behave. 
Figure 1 helps illustrate another important aspect of MRF models. To calculate the probability of events at some time $\mathrm{t}$, between event number $\mathrm{k}$ and event number $\mathrm{k}+1$, we may simply calculate the Bayesian convolution of $\mathrm{P}_{\mathrm{k}}{ }^{+}(\mathrm{X})$ and $\mathrm{P}_{\mathrm{k}+1}^{-}(\mathrm{X})$, where $\mathrm{P}^{-}$is computed by a backwards-time sequence of convolutions from node $\mathrm{N}$ to node $\mathrm{k}+1$ (the mirror image in time of equation 5). Again, this all follows easily from equation 3 . This reflects the basic Bayesian principle that the probability of something conditional on some external knowledge requires that we convolve what we know from all relevant sources of knowledge (prior to the actual run of the experiment), which includes knowledge about the initial conditions, knowledge about sources of noise during the experiment, and placement of things which affect the experiment at any time before it concludes.

In actuality, the choice of $t$, the line which slices figure 1, does not affect the prediction for $\operatorname{Pr}(\mathrm{X})$, in the MRF case. It is still the same set of possible outcomes, $\mathrm{X}$, whose probabilities we calculate, and the results are the same for any choice of $X$. This changes when we move on to consider paths $X(t)$, and when $\operatorname{Pr}^{+}$becomes a function of $X(t)$. In the case of stochastic path analysis, the Bayesian convolution from past and from future, at time $t$, gives probabilities for $\mathrm{X}(\mathrm{t})$.

\subsection{From $\mathrm{N}$ discrete events to long sequences in discrete time}

Stochastic path models may be developed as the limiting case of MRF models of long regular sequences of events $t_{k}$ from time $t_{-}$to $t_{+} \cdot A$ stochastic path model may be defined as the limit of such sequences, as $\Delta t=t_{k+1}-t_{k}$ goes to zero. In this case, we already assume that the path is a set of $X(t)$ over times $t$, with the Markov property. The Markov property can apply to Markov Random Fields, and not only Markov processes.

First let us review some basic properties of Markov chains, the case where the state $\mathrm{X}$ is just an integer $\mathrm{i}$ in the interval from 1 to N, and where locality (and space itself) is not considered. Such systems are often represented as:

$$
\operatorname{Pr}(j(t+1))=\sum_{k=1}^{n} M_{j k} \operatorname{Pr}(k(1))
$$

where

$$
\mathrm{M}_{\mathrm{jk}}=\operatorname{Pt}(\mathrm{j}(\mathrm{t}+1) \mid \mathrm{k}(\mathrm{t}))
$$

Some but not all Markov chains can be represented by an equivalent Markov chain running backwards through time. To have such a backwards time representation, a Markov chain M must have the property that the limit $\mathrm{p}^{*}$ of $\mathrm{M}^{\mathrm{T}} \mathrm{p}_{0}$ as $\mathrm{T}$ goes to infinity exists, is not dependent on the starting probability distribution $\mathrm{p}_{0}$, and has no zero elements. (Strictly speaking, the backwards representation also exists if the states of the system can be partitioned into groups of states such that there is never a transition from one group to another, and each group separately satisfies these criteria.) In that case, we can use Bayes' Law to deduce:

$$
\operatorname{Pr}(\mathrm{k}(\mathrm{t}) \mid \mathrm{j}(\mathrm{t}+1))=\left(\operatorname{Pr}\left(\mathrm{j}(\mathrm{t}+1) \mid \mathrm{k}(\mathrm{t}) \mathrm{p}_{\mathrm{k}}{ }^{*}\right) / \mathrm{p}_{\mathrm{j}}^{*}\right.
$$

Note that these conditional probabilities - the ones in forwards time and in backwards time - both embody a convolution of information from the dynamics within the time interval and from the boundary conditions $\mathrm{p} *$. Neither should be assumed to be suitable as a model of the ETSP, which should reflect only the conditions between time $t$ and time $\mathrm{t}+\Delta \mathrm{t}$.

In the more general case, where $\mathrm{X}$ is a more general kind of outcome, there are still transition probabilities underneath, and we may begin by considering a Markovian kind of model:

$$
\operatorname{Pr}(X(t+\Delta t))=\operatorname{Pr}(X(t))+\Delta t \int G(X, Y(t)) \operatorname{Pr}(Y(t) d Y(t)
$$

However, because of the nonlocal effects of the normalizations implied by equations 4, 5 and 8 , we cannot expect this direct Markov chain kind of representation to be local even when the underlying physical interactions (ETSP) are local, nor can we expect it to be time-symmetric (especially given how variable the boundary conditions $\mathrm{p}^{*}$ may be in infinite past or future time. Therefore, in the spirit of equation 5 , it is more suitable to represent the forward equations as:

$$
\operatorname{Pr}^{+^{*}}(X(t+\Delta t))=\operatorname{Pr}^{+}(X(t))+\Delta t \int G(X, Y(t)) \operatorname{Pr}^{+}(Y(t)) d Y(t)
$$




$$
\operatorname{Pr}^{+}(\mathrm{X}(\mathrm{t}+\Delta \mathrm{t}))=\mathrm{PR}^{+} *(\mathrm{t}+\Delta \mathrm{t}) / \mathrm{Z}(\mathrm{t}+\Delta \mathrm{t})
$$

We then ask that the core function which represents the ETSP be both local and time-symmetric:

$$
\mathrm{G}(\mathrm{X}, \mathrm{Y})=\mathrm{G}(\mathrm{Y}, \mathrm{X})
$$

except at times when positive time free energy is injected into the system. MRF1 and MRF3 each contain just one node where such injection takes place, the source of entangled photons; the modeling of that node is straightforward for purposes of these predictions (though one could go further and model the innards of a BBO crystal being pumped form a laser which then serves as the source, and so on). In principle, we should also add an exception for the case where negative time free energy is injected into the experiment, but no one on earth now seems to have the technology yet to complicate the predictions in that manner.

Equation 11 represents the Real Time Normalization of Probabilities (RTNP), which is an essential technical tool in practical stochastic path modeling. IN practice, however, since the product of such $\mathrm{Z}$ factors is still a scalar multiplier, we can use equation 10 to model what happens all across some interval of time (as as the time form when a photon enters a polarizer to when it leaves), and then do the normalization all at once across the entire interval, yielding equivalent results.

\subsection{Options for Continuous Time Models}

Section 3 of this paper will mirror the discussion of section 2.3 by giving an example of a stochastic path model for a polaroid polarizer which may be written in the form:

$$
\frac{d}{d t} \operatorname{Pr}^{+^{*}}(X)=\int G(X, Y) \operatorname{Pr}^{+}(Y) d Y
$$

and

$$
\frac{d}{d t} \operatorname{Pr}^{+}(X)=-Z(t) \operatorname{Pr}^{t}(X)+\int G(X, Y) \operatorname{Pr}^{+}(Y) d Y
$$

However, I will suppress the "+" superscript in section 3. G will obey the conditions of locality and time symmetry, as in equation 12 . Note that the symmetry constraint does not impinge on our freedom to model G(X,X), and we are emphatically not required to model $\mathrm{G}$ in a way which would result in conservation of probability without the use of RTNP as in equation 14. Indeed, if we chose a $G$ which does conserve probability without the use of Z, then the system would obey the axiom which Clauser et all called "causality" [1], and we would not be able to construct a local model capable of making the right ptedictions for Bell's Theorem experiments.

Equations 13 and 14 are convenient in general for the study of jump processes, like abrupt quantum transitions in which a photon may be absorbed or rotated abruptly to the preferred orientation of the polarizer. That is sufficient for the description of Bell's Theorem and triphoton experiments in which high quality polarizers are used, and effects like dissipation are suppressed. (For calcite polarizers the situation is even simpler, because of how the important effects occur precisely at the boundaries of a crystal.) However, pure jump models are not sufficient for modeling systems where effects like dissipation are important [14,15]. In that case, we may augment or replace equation 13 by some kind of Langevin equation [10,11], but still appending the crucial RTNP term in equation 14 . This suggests the interesting possibility that reverse-time dissipation may actually exist, under special circumstances in experiments like Bell or triphoton, and could possible be tested for.

Most of the work in the field of stochastic differential equations (including Langevin equations) has focused on cases where classical time-forwards causality is assumed, and formulated in a manner similar to what is used in [1]. There has been important work on the case of mixed causality, which turns out to be important in financial forecasting , but that work seems to have focused on special cases very different from the kinds of system relevant here. 


\section{Stochastic Path Model of Polaroid Polarizer CMRFp}

\subsection{Introduction}

The MRF1 model [2] has already provided a local realistic model giving correct predictions for Bell's Theorem experiments, based on an aggregate ETSP model of the input/output behavior of the polarizer. The MRF3 model provided more physical resolution for the case where the polarizer is a crystal polarizer, with properties dominated by the abrupt boundary conditions as light enters or leaves the crystal $[3,16]$. Here we will provide a stochastic path model for a different type of polarizer, within the same experiment. We will call this the CMRFp model, for continuous-time MRF model of polaroid polarizer.

The polaroid polarizer uses a different physical mechanism from that of the crystal polarizer [16]. Conceptually, it operates like a stack of tiny wires oriented parallel to the surface of the polarizer, Light may be absorbed or rotated by the first layer of wires, or by the second or the third. It moves steadily ahead from entry to exit or absorption. It is still true that light off of the preferred orientation may be absorbed or rotated within a millimeter or less (making it possible to build relatively thin polaroid glasses) but it is not an instantaneous surface effect, Unlike MRF3, CMRFp assumes that the dynamics are the same for each layer of wires (really, polymer fibers) which the light passes through, and that the layers are thin enough that we can model them as a continuum.

In [2] and [3], we write out the probabilities for all of the main possible outcomes $X$ of the Bell experiment as a whole. Here, we will only verify that CMRFp results in overall input/output behavior of the polarizer replicating the important properties of the MRF1 polarizer model which caused the correct predictions. The key property is that the Bayesian convolution predicted a physical picture similar to that of Klyshko in which retrocausal effects concentrated probability into scenarios where one of the entangled photons has a linear polarization identical to (or orthogonal to) the prefered direction of one of the polarizers, such that the double counting rate depends on what happens when the other photon reaches a polarizer tuned to the other angle. The same effects predict that probability in the triphoton experiment [2] will be concentrated in scenarios where two of the three photons match their respective polarizers (or are exactly orthogonal, resulting in the prediction of equation 1 , which differs substantially from that of traditional "collapse of the wave function" [2].

\subsection{Specification of the model}

CMRFp, like MRF3 [3], is intended to be the simplest possible model giving a plausible first order picture of what is happening inside one type of polarizer in a Bell or triphoton experiment. Because there is only one photon entering each polarizer from the source of entangled photons, CMRFp simply characterizes the fate of a single photon as it propagates between the source and the detectors.

The photon is assumed to be in any one of three possible states, which we will label as " $\{0\}$ " (absence of a photon), " $\theta_{\mathrm{a}}$ " (a photon with the angle of linear polarization preferred by whichever polarizer is currently under analysis), and " $\theta$ ", and other orientation between $\theta_{\mathrm{a}}$ and $\theta_{\mathrm{a}}+\pi / 2$. More precisely, the set of possible states includes the two points $\{0\}$ and $\theta_{\mathrm{a}}$ and the open interval of possible values of $\theta$ between $\theta_{\mathrm{a}}$ and $\theta_{\mathrm{a}}+\pi / 2$, and we assume that these are enough to mirror the dynamics of all possible linear polarizations in practice due to the underlying symmetries.

Because CMRFp is intended, above all, to offer a simple understandable example of the more general methods of stochastic path modeling, we chose not to add terms to describe circular polarization, as we did with MRF3 [3], where it was found that those terms did not really affect the resulting predictions, for reasons which would apply here as well.

In the case of crystal polarizers, we knew that boundary conditions for light are the decisive cause of polarization [3], and there was not much choice of model. Here, it is conceivable that a diffusion type model [13] or a hybrid jump-diffusion model might also have the right properties to correctly predict Bell experiments, and be equally

plausible, but we have not yet worked on developing such an alternative. Also, for polaroid polarizers in this kind of experiment, we may assume that the photon enters the polarizer at a definite time $t_{\text {. }}$ and leaves (or is absorbed by) at another definite time $t_{+}$.

The core equations for CMRFp proper, a special case of equation 13, start with two definitions:

$$
\mathrm{G}^{+}(\theta)=\alpha \cos ^{2}\left(\theta-\theta_{\mathrm{a}}\right)+\mathrm{g}_{0} \mathrm{~g}\left(\theta-\theta_{\mathrm{a}}, \mathrm{W}\right)
$$


$G^{-}(\theta)=\beta \alpha \sin ^{2}\left(\theta-\theta_{a}\right)+\beta g_{0} g\left(\pi / 2+\theta_{a}-\theta, W\right)$

and the following three dynamic equations for the times when the photon is in the polarizer (i.e., between $t_{-}$and $t_{+}$):

$$
\begin{aligned}
& \frac{d}{d t} \operatorname{Pr}^{*}(\theta)=-\gamma \operatorname{Pr}^{*}(\theta)+G^{+}(\theta) \operatorname{Pr}^{*}\left(\theta_{a}\right)+G^{-} \operatorname{Pr}^{*}(\{0\}) \\
& \frac{d}{d t} \operatorname{Pr}^{*}\left(\theta_{a}\right)=-\gamma_{1} \operatorname{Pr}^{*}\left(\theta_{a}\right)+\int_{0}^{\pi} G^{+}(\theta) \operatorname{Pr}^{*}(\theta) d \theta \\
& \frac{d}{d t} \operatorname{Pr}^{*}(\{0\})=-\gamma_{2} \operatorname{Pr}^{*}(\{0\})+\int_{0}^{\pi} G^{-}(\theta) \operatorname{Pr}^{*}(\theta) d \theta
\end{aligned}
$$

The most important parameters of the model are $\gamma, \alpha$ and $\beta$, but $g_{0}, \gamma_{1}$ and $\gamma_{2}$ are included for purposes of discussion (and possible future use). The function $\mathrm{g}(\mathrm{x}, \mathrm{W})$ is a regularization function, to approximate the Dirac delta $\delta(\mathrm{x})$ in the limit as W goes to infinity. In MRF1, we used a simple delta function $\delta$, but the model and its predictions are more rigorously defined when we define them as a limit in this way.

Intuitively, $\alpha$ represents the probability of rotation of the photon, $\beta$ represents the probability of absorption or emission of a photon (which happens "on top" of rotation to an angle form which absorption is possible), the $\gamma$ term represents the decay of photons not aligned either at or perpendicular to the direction of the fibers which this kind of polarizer is made of, and g represents the thermodynamic pressure of the polarizer to favor the preferred orientation and let it propagate through. For a deeper understanding of $\alpha$ and $\beta$, consider the same parameters in the MRF3 model [3]. These equations were the simplest possible equations able to replicate the main features of the ETSP of the MRF1 model of a polarizer [2]. Both parameters, $\alpha$ and $\beta$, are assumed to be small, representing the fact that very little would happen at all in these experiments were it not for the strong impact of the source of entangled photons.

The equations in this form fully satisfy the symmetry requirement (equation 12) in the limit as $\mathrm{W}$ goes to infinity, if we remember that the terms for outflux from $\{0\}$ and $\theta_{\mathrm{a}}$ to $\theta$ are proportional to the probabilities of $\{0\}$ and $\theta_{\mathrm{a}}$, and hence subsumed in $\gamma_{1}$ and $\gamma_{2}$. However, for an ideal polarizer (and a good approximation to high quality realistic polarizers used in Bell experiments), we may simply take $\gamma_{1}$ and $\gamma_{2}$ to be zero, and delete the unnecessary terms. The $\gamma$ parameter proper can be seen intuitively as "the probability that the photon decides it didn't want to be here in the first place," and retroactively reducing its probability of being there; it plays an essential role here.

To fully satisfy time-symmetry (equation 12) for finite W, we should add terms into equation 17 to reflect the action of "g" in creating new photons near to, but distinct from, $\theta_{\mathrm{a}}$ and $\theta_{\mathrm{a}}+\pi / 2$. However, as W goes to infinity, these terms basically just modify the values of $\gamma_{1}$ and $\gamma_{2}$, which are model parameters in any case. It is enough to satisfy timesymmetry for the model in the limit as $\mathrm{W}$ goes to infinity.

For a more complete statement of the model, we remember that this photon is just one part of an entangled system, whose state at time $t$ is $X(t)$. Let us use "R(t)" (or just $R$, with "t" understood) to denote the state of the rest of the system, the state of the other photons in the case of Bell or triphoton experiments. In that case, for times when the other photons are propagating in free space, the full dynamic equations of the model, corresponding to equation 13, are:

$$
\begin{aligned}
& \frac{d}{d t} \operatorname{Pr}^{+}(\theta, R)=-\gamma \operatorname{Pr}^{+}(\theta, R)+G^{+}(\theta) \operatorname{Pr}^{+}\left(\theta_{a}, R\right)+G^{-} \operatorname{Pr}^{+}(\{0\}, R)-Z \operatorname{Pr}^{+}(\theta, R) \\
& \frac{d}{d t} \operatorname{Pr}^{+}\left(\theta_{a}, R\right)=-\gamma_{1} \operatorname{Pr}^{+}\left(\theta_{a}, R\right)+\int_{0}^{\pi} G^{+}(\theta) \operatorname{Pr}^{+}(\theta, R) d \theta-Z \operatorname{Pr}^{+}\left(\theta_{a}, R\right)
\end{aligned}
$$




$$
\frac{d}{d t} \operatorname{Pr}^{+}(\{0\}, R)=-\gamma_{2} \operatorname{Pr}^{+}(\{0\}, R)+\int_{0}^{\pi} G^{-}(\theta) \operatorname{Pr}^{+}(\theta, R) d \theta-Z \operatorname{Pr}^{+}(\{0\}, R)
$$

Because we assume locality here (as in the MRF models), there is no real interaction with the rest of the system, except by way of the global partition factor $Z(t)$. We may calculate the overall predictions that light first enters and leaves (or is absorbed) by one polarizer, then another, without changing the result from what is predicted when they are all entered at the same time. This contrasts sharply with predictions based on collapse of the wave function [2], which is a highly nonlocal model now under test.

\subsection{Predictions of the Model}

\section{3,3,1 First order terms in $\operatorname{Pr}^{*}\left(t_{+}\right)$}

This subsection will calculate the first order terms in the perturbation expansion to predict $\operatorname{Pr}^{+*}\left(\right.$ state, $\left.t_{+}\right)$for those cases

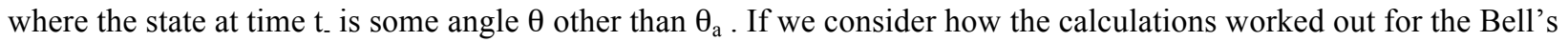
Theorem experiment in [2] and in [3], we can see that the only terms in the perturbation expansion which contribute to the prediction are terms on the order of $\alpha^{0}$ or $\alpha^{1}$; the others make small contributions, because $\alpha$ itself is assumed to be small. (Still, the previous calculations also show that we should retain terms on the order of $\alpha \beta$, even though $\beta$ is also small, because of what happens later when we consider the detectors, as discussed most completely in [3].)

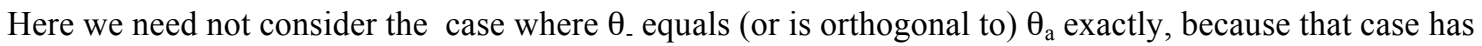
probability measure zero in the probability distribution emerging from the sources. That is true for all values of $\mathrm{W}$, and thus for the limit as $\mathrm{W}$ goes to infinity as well.

We believe that any reasonable regularization function $\mathrm{g}$ approaching $\delta$ would lead to the same predictions (limit as W goes to infinity). However, for convenience here, we further specify the theory by choosing the regularization function:

$$
\begin{aligned}
g(x, W) & =W \text { when }|x|<\frac{1}{2 W} \\
& =0 \text { otherwise }
\end{aligned}
$$

We assume that the polarizer has high enough quality that virtually all of the light in the preferred direction $\left(\theta_{a}\right)$ goes through, while only a negligible fraction of the photons of other polarizations simply pass through the polarizer. This fits with the more detailed discussion of polarizer quality by Clauser and Shimony[1]. This is why it makes sense to ignore the $\gamma_{1}$ and $\gamma_{2}$ terms in these calculations, and to assume that the zeroth order terms in the perturbation expansion

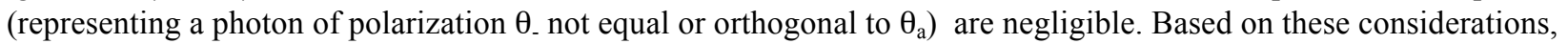
we need only consider those terms in the perturbation expansion which account for one transition, from $\theta_{-}$to $\theta_{\mathrm{a}}$ or to $\{0\}$, based on the terms in equations 18 and 19 which represent that kind of transition.

Under these assumptions, and under the regularization shown in equation 3, we get different predictions for $\operatorname{Pr}^{*}\left(t_{+}\right)$for the three possibilities for $\theta_{-}:(1)$ "near $\theta_{a}$," meaning that $\theta_{a}+1 / W>\theta_{-}>\theta_{a} ;$; (2) "near orthogonal," meaning that $\theta_{\mathrm{a}}+\pi / 2>\theta_{-}>\theta_{\mathrm{a}}+\pi / 2-1 / \mathrm{W}$; and (3) normal middle cases, where $\theta_{\mathrm{a}}+\pi / 2-1 / \mathrm{W}>\theta_{-}>\theta_{\mathrm{a}}+1 / \mathrm{W}$. Applying first order perturbation theory to equations 17 and 18 , for the normal middle cases we arrive at:

$$
\begin{gathered}
\operatorname{Pr}^{+^{*}}\left(\theta_{a}\left(t_{+}\right) \mid \theta_{-}\left(t_{-}\right)\right) \approx \alpha \cos ^{2}\left(\theta_{a}-\theta_{-}\right) \int_{t_{-}}^{t} e^{-\gamma\left(t-t_{-}\right)} d t \\
=\frac{\alpha \cos ^{2}\left(\theta_{a}-\theta_{-}\right)}{\gamma}<\frac{\alpha}{\gamma}
\end{gathered}
$$

Applying first order perturbation theory to equations 17 and 19, we arrive at: 


$$
\begin{gathered}
\operatorname{Pr}^{+^{*}}\left(\{0\}\left(t_{+}\right) \mid \theta_{-}\left(t_{-}\right)\right) \approx \beta \alpha \sin ^{2}\left(\theta_{a}-\theta_{-}\right) \int_{t_{-}}^{t_{-}} e^{-\gamma\left(t-t_{-}\right)} d t \\
=\frac{\beta \alpha \sin ^{2}\left(\theta_{a}-\theta_{-}\right)}{\gamma}<\frac{\beta \alpha}{\gamma}
\end{gathered}
$$

For the case of $\theta_{-}$near $\theta_{a}$, equation 25 still applies, but, because $\mathrm{G}^{+}$reflects the regularization, equation 24 changes to:

$$
\begin{gathered}
\operatorname{Pr}^{+*}\left(\theta_{a}\left(t_{+}\right) \mid \theta_{-}\left(t_{-}\right)\right) \approx\left(\alpha \cos ^{2}\left(\theta_{a}-\theta_{-}\right)+g_{0} W\right) \int_{t_{-}}^{t} e^{-\gamma\left(t-t_{-}\right)} d t \\
=\frac{\alpha \cos ^{2}\left(\theta_{a}-\theta_{-}\right)+g_{0} W}{\gamma} \approx \frac{\alpha+g_{0} W}{\gamma}
\end{gathered}
$$

Likewise, for near orthogonal cases, equation 24 still applies, but in place of equation 25 we have:

$$
\begin{gathered}
\operatorname{Pr}^{+^{*}}\left(\{0\}\left(t_{+}\right) \mid \theta_{-}\left(t_{-}\right)\right) \approx\left(\beta \alpha \sin ^{2}\left(\theta_{a}-\theta_{-}\right)+\beta g_{0} W\right) \int_{t_{-}}^{t} e^{-\gamma\left(t-t_{-}\right)} d t \\
=\frac{\beta \alpha \sin ^{2}\left(\theta_{a}-\theta_{-}\right)+g_{0} W}{\gamma} \approx \frac{\beta \alpha+\beta g_{0} W}{\gamma}
\end{gathered}
$$

\subsubsection{Overall Predictions for Bell and Triphoton Experiments}

Equations 24 through 27 give the predictions of the CMRFp model for the overall input/output behavior of a polaroid polarizer in a Bell's Theorem or triphoton experiment, or similar circumstances. In order to predict the actual counting rates, $R_{2} / R_{0}$ and $R_{3} / R_{0}$, for these experiments, we simply need to insert these equations into the greater MRF calculations given in more detail in [2] and in [3].

For the Bell's Theorem experiment, we use the picture given in Figure 2. In this picture, the $\theta$ variables represent linear polarization of a photon on the left and right channels, before and after the polarizers. The $\gamma$

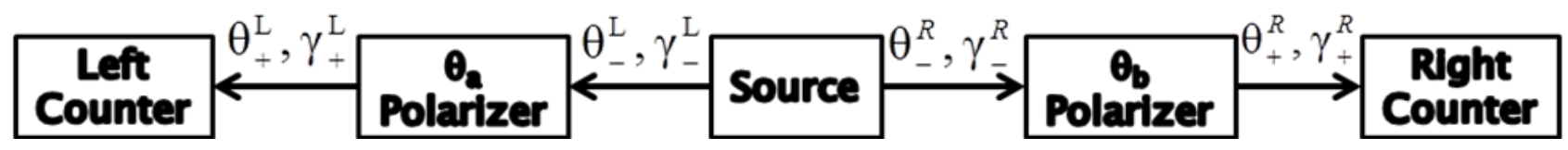

Figure 2. The original Bell's Theorem experiments [1,2]

variables are logical variables, representing presence or absence of a photon. There are five nodes in the graph. The task is to predict $\operatorname{Pr}\left(\gamma_{+}{ }^{\mathrm{L}}, \gamma_{+}{ }^{\mathrm{R}}\right)$ in situations where two entangled photons are emitted from the source $\left(\right.$ when $\left.\gamma_{-}^{\mathrm{L}}=\gamma_{-}{ }^{\mathrm{R}}=1\right)$. We can make this prediction using the MRF methods discussed in section 2. Because we are aiming to predict probabilities for detections at the final time, we can do this simply by marching forwards in time, as in equations 20, 21 and 22 Because the Pr* calculations on the left channel are independent from those on the right channel (except for the need to normalize total probabilities to one), we can charge forwards on the left, and then on the right, and normalize probabilities to add up to one at the end.

The time-forwards calculations begin with the same simple but adequate model of the source used in [2] and [3], which may be written as:

$$
\begin{aligned}
& \operatorname{Pr}^{+}\left(\theta_{-}\right)=(1 / \pi) d \theta_{-} \\
& \theta_{-}{ }^{\mathrm{L}}=\theta_{-}{ }^{\mathrm{R}}=\theta_{-}
\end{aligned}
$$


Because probabilities will be normalized to one, in the end, we can simplify the calculations by deleting the factor of $1 / \pi$, which will not change the predictions.

Next convolve this with the polarizer on the left, using equations 24 through 27 but accounting for the larger

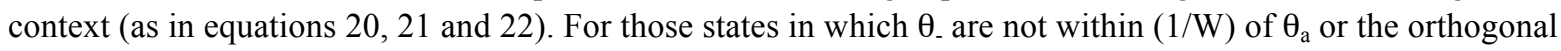
direction, we obtain:

$$
\begin{aligned}
& \operatorname{Pr}^{+*}\left(\theta_{a}\left(t_{+}\right), \theta_{-}\right)=\frac{\alpha \cos ^{2}\left(\theta_{a}-\theta_{-}\right)}{\gamma} d \theta_{-} \\
& \operatorname{Pr}^{+^{*}}\left(\{0\}\left(t_{+}\right), \theta_{-}\right)=\frac{\beta \alpha \sin ^{2}\left(\theta_{a}-\theta_{-}\right)}{\gamma} d \theta_{-}
\end{aligned}
$$

For those states $\theta_{\text {. near }} \theta_{\mathrm{a}}$, equation 31 still applies, but equation 30 is replaced by:

$$
\operatorname{Pr}^{+*}\left(\theta_{a}\left(t_{+}\right), \theta_{-}\right)=\frac{\alpha \cos ^{2}\left(\theta_{a}-\theta_{-}\right)+g_{0} W}{\gamma} d \theta_{-}
$$

Likewise, for near orthogonal cases, equation 30 still applies, but equation 31 is replaced by:

$$
\operatorname{Pr}^{+^{*}}\left(\{0\}\left(t_{+}\right), \theta_{-}\right)=\frac{\beta \alpha \sin ^{2}\left(\theta_{a}-\theta_{-}\right)+\beta g_{0} W}{\gamma} d \theta_{-}
$$

Finally, to complete the convolution along the left channel, we convolve with the simple but adequate model of the detectors in [2] and [3]. In that model, the detectors are assumed to be perfectly efficient, but the $\mathrm{p}^{*}$ node probability is 1 for the event of no detection and $\beta$ for the event of detection. Applying this to equations 30 to 33, we get first the middle cases:

$$
\begin{aligned}
& \operatorname{Pr}^{+*}\left(\gamma_{+}^{L}, \theta_{-}\right)=\frac{\beta \alpha \cos ^{2}\left(\theta_{a}-\theta_{-}\right)}{\gamma} d \theta_{-} \\
& \operatorname{Pr}^{+*}\left(N O T \gamma_{+}^{L}, \theta_{-}\right)=\frac{\beta \alpha \sin ^{2}\left(\theta_{a}-\theta_{-}\right)}{\gamma} d \theta_{-}
\end{aligned}
$$

For values of $\theta_{-}$near $\theta_{\mathrm{a}}$, equation 34 is replaced by:

$$
\operatorname{Pr}^{+*}\left(\gamma_{+}^{L}, \theta_{-}\right)=\frac{\beta \alpha \cos ^{2}\left(\theta_{a}-\theta_{-}\right)+\beta g_{0} W}{\gamma} d \theta_{-}
$$

For near orthogonal values, equation 35 is replaced by:

$$
\operatorname{Pr}^{+^{*}}\left(N O T \gamma_{+}^{L}, \theta_{-}\right)=\frac{\beta \alpha \sin ^{2}\left(\theta_{a}-\theta_{-}\right)+\beta g_{0} W}{\gamma} d \theta_{-}
$$

Notice that we can divide by $\beta$ and by $g_{0}$ and multiply by $\gamma$, without changing what we will get later when we normalize all probabilities to add up to one. After we do that, the total probabilities for the middle values of $\theta_{\text {. }}$ (equation 34 plus

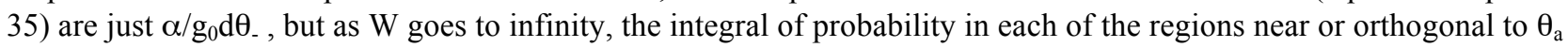


goes to one. Under our assumption that $\alpha$ is a very small number, this implies that the photon emitted in the beginning will have a very high probability of being either aligned with $\theta_{\mathrm{a}}$ or orthogonal to it. It seems as if the polarizer reached back in time to make sure something is emitted which it can either absorb or pass on easily.

However, this picture changes after we do yet another convolution, up the right channel. So long as $\theta_{\mathrm{b}}$ does not

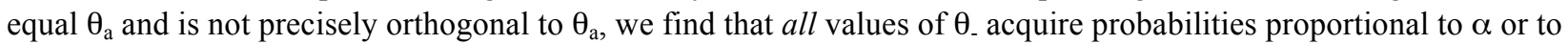
$\alpha^{2}$. The values which are not close to $\theta_{\mathrm{a}}$ or $\theta_{\mathrm{b}}$ or orthogonal to them are of order $\alpha^{2}$, and do not contribute to the prediction, exactly as before [3]. Thus we only need to analyze four cases. In the case where $\theta_{-}=\theta_{-}{ }^{\mathrm{R}}=\theta_{\mathrm{a}}$, a photon will always be detected on the left side, and the probability of a double count is simply the same as the usual probability of a photon of orientation $\theta_{\mathrm{a}}$ making it through a polarizer of orientation $\theta_{\mathrm{b}}$, i.e. $\cos ^{2}\left(\theta_{\mathrm{a}}-\theta_{\mathrm{b}}\right)$. The same result obtains, in mirror image, in the case when $\theta_{-}=\theta_{-}^{\mathrm{L}}=\theta_{\mathrm{a}}$. The two orthogonal cases have the same probability as the two near cases, but there is no chance of a double count in those cases; thus, when we consider them, we replicate the standard wellverified predictions for the Bell experiment:

$$
\mathrm{R}_{2} / \mathrm{R}_{0}=(1 / 2) \cos ^{2}\left(\theta_{\mathrm{a}}-\theta_{\mathrm{b}}\right)
$$

For the new triphoton experiment, the graph of the experiment is slightly more complicated, as shown in Figure 3, and notation changes slightly.

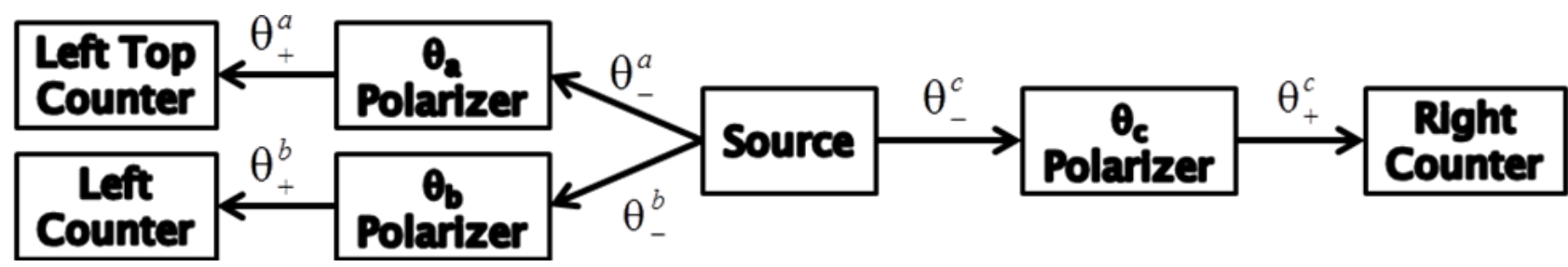

Figure 3. The triphoton experiment proposed in [2,3]

The source is a source of three entangled photons, represented by the wave function:

$$
\psi=\frac{1}{\sqrt{2}}\left[|0\rangle_{a}|0\rangle_{b}\left|\frac{\pi}{2}\right\rangle_{c}+\left|\frac{\pi}{2}\right\rangle_{a}\left|\frac{\pi}{2}\right\rangle_{b}|0\rangle_{c}\right]
$$

We may model this source as :

$$
\begin{aligned}
& \operatorname{Pr}^{+}\left(\theta^{\mathrm{a}}\right)=(1 / \pi) d \theta^{\mathrm{a}}{ }_{-} \\
& \operatorname{Pr}^{+}\left(\theta^{\mathrm{b}}\right)=(1 / \pi) d \theta^{\mathrm{b}}{ }_{-} \\
& \theta_{-}^{\mathrm{c}}=\theta_{-}^{\mathrm{a}}+\theta_{-}^{\mathrm{b}} \quad(\bmod \pi)
\end{aligned}
$$

The convolutions on the three channels work out essentially the same way, except that the normalized probabilities are dominated by cases in which two of the three photons exactly match (or are exactly orthogonal to) the preferred orientations of the polarizers they go to. For example, in the case where $\theta^{a}=\theta_{a}$ and $\theta^{b}=\theta_{b}$, photons will be detected on the $\mathrm{a}$ and $\mathrm{b}$ channels, and the three-photon coincidence rate is determined by the rate at which a photon of polarization $\theta^{c}=\theta_{a}+\theta_{b}$ passes through a polarizer of orientation $\theta_{c}$. That rate is $\cos ^{2}\left(\theta_{c}-\theta_{a}-\theta_{b}\right)$. We get the same rate for both of the other positive cases. There are, again, no triple counts in cases where one of the photons is orthogonal to the polarizer it goes to. As with the Bell's Theorem example, we arrive at a simple prediction, the prediction shown in equation 1.

\section{Possibilities for Future Research}

There are two main possibilities for further research to build on these findings.

First is the use of the stochastic path method, as described in section 2 and illustrated in section 3, to predict and understand other experiments beyond the Bell and triphoton experiment. 
The most obvious place to look for new experiments lies in the realm of technologies which use spin gates to select preferred polarizations, either of photons or of other particles such as electrons or polaritons. These are basically just polarizers by another name. Many of these spin gates, like the new digital spin switch for photons developed by the Joint Quantum Institute at Maryland, can only be tuned "up" or "down," because they were designed to implement the traditional paradigm of quantum computing developed by David Deutsch relying on qubits $(\mid 1>$ or $\mid 0>)$. The types of models described here would be overkill for such work. However, recent work from several groups $[17,18]$ has shown that there can be substantial benefits to secure quantum communication by broadening the paradigm, to consider spin gates which are tunable over continuous angles. When we want to build systems which involve complex chains of polarizers processing entangled photons, it will be important to model the polarizers in a physically realistic way, which does not entail unitary dynamics in objects like polarizers. Hopefully, the new triphoton experiment will validate either stochastic path models or collapse of the wave function (as described in the appendix of [2]), which can then be used as a basis for design in this space. Tunable spin gates will also be important to forms of quantum computing using spintronics or spins of polaritons. On the empirical side, Bell's Theorem and triphoton experiments using nanotechnology could be useful in clarifying these connections.

Beyond polarizers, it would also be interesting to explore the use of stochastic path models in other traditional areas of quantum optics, such as the study of antibunching. Based on the review of H. Paul [3], it seems likely that classical stochastic models representing the photon as a particle rather than a wave may already be enough to describe many experiments of that type, but when they are linked to entanglement in nonclassical ways, this new description may be useful.

In general, much of the existing work in quantum optics $[13,14]$ shows that models assuming discrete sets $\mathrm{X}(\mathrm{t})$ of state variables can be powerful enough to describe many situations. Work in quantum field theory, using Feynman path methods [20], shows that graphical methods can be useful in a very wide range of circumstances.

Second, however, there is a possibility that a more radical extension of section 2 might be both workable and useful. There are three obvious logical possibilities for trying to extend stochastic path methods to the case where the state $\mathrm{S}(\mathrm{t})$ at time $\mathrm{t}$ is defined by fields over continuous space:

(1) The mainstream approach, where stochastic path methods are used to calculate probability distributions for wave functions, presumably represented by density operators such as:

$$
\rho(t)=\int \operatorname{Pr}(\alpha) \psi_{\alpha}(t) \psi_{a}^{H}(t) d \alpha,
$$

where each $\psi_{a}$ is a full function of Fock space obeying the usual Schrodinger equation of canonical quantum field theory $[8,9,20]$ :

$$
\dot{\psi}=i h \psi
$$

(2) The functional integral approach, where we extend equation 3 in the same way that Feynman and Schwinger extended equation 2 long ago [20,21], by trying to define:

$$
\operatorname{Pr}(\{X(\underline{x}, t)\})=\frac{1}{Z} e^{-\iiint \int L\left(X(\underline{x}, t) d^{3} \underline{x} d t\right.},
$$

where we now use $X(\underline{x}, t)$ to represent the set of field values at the point $(\underline{x}, t)$ in space-time;

(3) The neoclassical approach, where stochastic path methods are used to calculate probabilities of the state of

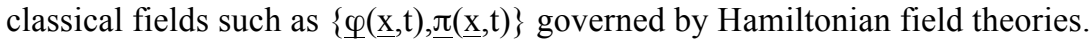

Traditional canonical quantum field theory (KQFT) combined equation 44 with assumptions such as the collapse of the wave function to generate predictions. It is interesting that Hugh Everett, the founder of the many-worlds version of quantum theory, tried to derive measurement assumptions such as collapse of the wave function, from equation 44, but was not really able to establish a clear connection [9]. The challenge here is to follow up on the ideas in [6] to develop a new mainstream formalism.

In all three cases, we would propose a two or three stage effort to develop a practical tool for use in prediction. Ideally, this could be done in detail for all three approaches, and tested by experiment, rather than selected by cultural prejudice or political vote. The first stage is to predict the equilibrium probabilities for a universe governed by each of these possibilities. This is essentially just a matter of closed systems thermodynamics, where we view the universe itself as a closed system. A second possible stage is to evaluate various cross-covariance functions, but we will not discuss that here. The third stage would be to use the equilibrium probabilities as the boundary conditions for infinite future and past time, but use stochastic path analysis to fill in probabilities between a set of discrete times, from $t_{1}$ to $t_{N}$, at which 
the experimenter intervenes somehow to create a discrete transition. The discrete transition may simply be a matter of entering or exiting a polarizer, as in the example of section 3, or it may be an active boundary condition (as in [3]), or it may be the kind of quantum transition described by Carmichael in his discussion of quantum trajectory simulation [22]. The third stage addresses the case of those open systems in which energy may be injected from the outside, or noise may enter from "reservoirs" (like polarizers) which are not modeled in detail at the quantum level.

In all three cases, we would address the first stage in two steps. First, identity a basic invariant measure, a measure dS such that the integral of $\operatorname{Pr}(\mathrm{S})$ in any region remains constant under the dynamics of the system. Such an invariant measure would already be an equilibrium probability measure, if it were a probability measure at all; however, to be an invariant probability measure, it must not only be an invariant measure, but must also have the crucial property that probabilities add up to one. Thus the second step is simply to multiply the measure dS by any function fof the conserved quantities of the system (also unchanged by the dynamics) which results in a finite integral, which may be normalized to 1 . Any such distribution is a valid possible equilibrium probability distribution. Thermodynamics usually goes further, to select particular equilibria of interest, but here we will not go that far.

For the mainstream approach above, the first stage is relatively easy. From equation 44, it is easy to see that $\rho_{0}=\mathrm{I}$ is an invariant distribution. Thus we easily arrive at a traditional type of distribution:

$$
\rho=\frac{1}{Z} f(H, \underline{P}, \underline{Q}, \ldots)
$$

for any appropriate function $\mathrm{f}$, such as the usual grand canonical Boltzmann distribution of Quantum Statistical Thermodynamics (QDT):

$$
\rho=\frac{1}{Z} \exp \left(-k_{0} H-k_{1} P_{1}-k_{2} P_{2}-\ldots\right)
$$

In principle, we must also address how to handle noncommuting conserved quantities, but in general, the situation here is well within the scope of traditional QST. Here we assume that "f" and "exp" are analytic functions of their operator arguments, and that the functions are defined by mapping ordinary multiplication into operator multiplication, as is required to ensure that such functions of conserved quantities are themselves conserved quantities under equation 44 .

The second stage is also straightforward, in situations like section 3 where the outcome is not really influenced by conditions beyond the final transition time $\mathrm{t}_{\mathrm{N}}$. We can apply forward time-marching, as in equations 5 and 14 , but only if we develop time-symmetric field models for the evolution of $\rho$ in all time intervals and all transition times of the experiment, except for the times when we inject positive time free energy into the system. Thus in effect we have a modeling framework here, where we still need to develop transition models and master equations $[13,14]$ within the constraints discussed above in order to make predictions. There are also some types of experiments [23] where more detailed modeling of the forwards time boundary conditions is essential, and there is an issue of how to do Bayesian convolution between two density operators, one from the past and one from the future.

Next, the functional integral approach is more difficult here. Equation 45 does not result in a conventional kind of stochastic partial differential equation (PDE), because the "white noise" implicitly assumed for every point in spacetime would not in general satisfy the usual assumptions about correlations across time embedded in Wiener processes or Langevin equations $[10,12]$. The neoclassical approach is essentially just the special case of the functional integral approach, in the limit as the magnitude of the noise goes to zero. It would be worthwhile, in principle, to study the range of possible predictions of the general case, but the neoclassical case is an easier starting point, and we do not yet see evidence for the noise to be nonzero[24]. The neoclassical approach basically embodies the idea that apparent white noise can arise as the result of emergent statistics due to nonlinearities and chaos. Stochastic distributions both from past and future, and from reservoirs traveled through, appear to be enough for now to explain the observed stochastic effects.

For the neoclassical approach, we have a simple but important new result to report here, related to the first stage of the proposed program. In the appendix of [15], we defined a very broad class of Hamiltonian field theories which we called "statistically incompressible" (SI) field theories. For this class, we found previously that there is a simple invariant measure:

$$
\mathrm{ds}=\mathrm{d}^{\mathrm{n}} \varphi \underline{\varphi} \mathrm{d}^{\mathrm{n}} \cdot \underline{\pi} \quad,
$$

where " $\mathrm{n}$ " is the number of components of $\varphi$ (and, of course, of $\pi$ ) and where the infinity reminds us that this is a measure for functions over space with an infinite number of points. The new result is that when we insert this invariant 
measure into the definition of $\rho$ (as in equations 8, 14 and 22 of [15]) in my extended P mapping, and perform the integration, it turns out that $\rho_{0}=\mathrm{I}$ is exactly the density operator corresponding to this measure. The integration is straightforward but tedious. This results in equilibrium distributions analogous to equations 46 and 47 :

$$
\begin{aligned}
& \rho=\frac{1}{Z} f_{N}(H, \underline{P}, \underline{Q}, \ldots) \\
& \rho=\frac{1}{Z} \exp _{N}\left(-k_{0} H-k_{1} P_{1}-k_{2} P_{2}-\ldots\right)
\end{aligned}
$$

but with one important difference, indicated by the "N" subscript. In this case, the operators H, P and Q all refer to normal form operators (as discussed in [15]) and the multiplications implicit in the analytic functions $f$ and exp are mapped into operator normal products rather than ordinary simple matrix multiplication. Still, equations 49 and 50 provide a density matrix representation, a simple "primal" representation, unlike the trickier dual representations discussed only briefly in the appendix of [15]. Equations 49 and 50 would imply a small change in the initial time boundary conditions for stage 2 , but still call for time-symmetric master equations and transition models in modeling experiments.

An interesting property of these distributions is that they can be well defined even for energy operators which are not strictly positive definite, if they are at least bounded from below, at least in analyses of universes with topology of a rectangular torus. It would be interesting to see whether they would have value in characterizing patterns such as cells which might emerge, statistically, for some field theories, where the ambient medium has positive energy and certain structures might have at least locally minimum energy.

More seriously, both for the functional integral approach and the neoclassical approach, the method clearly seems suitable for bosons (any bosons, really, as the generalized P mapping applies to them), but not to fermions. In fact, equation 2, representing the Feynman path approach, has the same problem. Historically [20] this has been addressed by introducing mechanisms like "ghosts" in implementing the field theories, or "anticommuting real numbers" [21], which could possibly work here as well. Alternatively, fundamental fermions might be modeled as topological solitons; extensive research at many places on "bosonization" [25] has shown how the statistics of such solitons may be fermionic, even when the underlying fields are themselves bosonic. The choice between these options certainly is a matter for future research, even in the Feynman path approach itself.

\section{References}

[1] Clauser, J.H. and Shimony, A., "Bell's Theorem: experimental tests and implications," Rep. Prog. Phys,Vol. 41, 1978, p.1881-1927.

[2] Werbos, P.J, "Example of lumped parameter modeling of a quantum optics circuit," SPIE Proc. Quantum Information and Computation XII, May 2014 SPIE 9123-10, http://arxiv.org/abs/1309.6168

[3] Werbos, P.J., , Local realistic model of Bell Theorem experiment and alternative model of quantum measurement. arXiv:1403.0469

[4] Shih, Yanhua, New lower-cost technology to produce many entangled photons and test their properties, NSF, http://www.nsf.gov/awardsearch/showAward?AWD_ID=1444491

[5] Peng, Tao, Hui Chen, Yanhua Shih, and Marlan O. Scully. "Delayed-Choice Quantum Eraser with Thermal Light." Physical review letters 112, no. 18 (2014): 180401.

[6] Werbos, P.J. "Bell's theorem, many worlds and backwards-time physics: not just a matter of interpretation." International Journal of Theoretical Physics 47.11 (2008): 2862-2874.

[7] Bell, J.S., The Speakable and Unspeakable in Quantum Mechanics, Cambridge U. Press, 1987

[8] Deutsch, David, The Fabric of Reality: The Science of Parallel Universes and Its Implications, Penguin, 1997

[9] DeWitt, B.S. \& Graham, N. eds, The Many Worlds Interpretation of Quantum Mechanics, Princeton U. Press, 1973

[10] Oksendal, Berndt, Stochastic Differential Equations: An Introduction with Applications. Fifth Edition, Corrected Printing, Springer-Verlag Heidelberg New York

[11] Kwok, Sau Fa. "Langevin equation with multiplicative white noise: Transformation of diffusion processes into the Wiener process in different prescriptions." Annals of Physics 327, no. 8 (2012): 1989-1997.

[12] N.El-Karoui \& L.Mazliak, eds, Backward stochastic differential equations, Addison-Wesley Longman, 1997.

[13] Carmichael Howard J. Carmichael, Statistical Methods in Quantum Optics1: Master Equations and Fokker-Planck 
Equations. Berlin: Springer, 1999.

[14] D.F.Walls and G.F. Milburn, Quantum Optics. New York: Springer, 1994.

[15] Werbos, P., "Extension of the Glauber-Sudarshan mapping for classical and quantum energy spectra." International IFNA -ANS Journal "Problems of nonlinear analysis in engineering systems", http://www.kcn.ru/tat_en/science/ans/journals (2014). arXiv:1402.5116

[16] S.G. Lipson, H. Lipson and D.S. Tannhauser, Optical Physics, Third Edition. Cambridge University Press, 1995

[17] Bhagyashri A. Darankur and Pramode K. Verma, "The braided single-stage protocol for quantum-secure communication," SPIE Proc. Quantum Information and Computation XII, May 2014

[18] Hong Lai, Mehmet Orgun, Liyin Xue, Jinghua Xiao and Josef Pieprzyk, "Dual compressible hybrid quantum secret sharing schenes based on extended unitary operation," SPIE Proc. Quantum Information and Computation XII, May 2014

[19] Paul, H. "Photon antibunching." Reviews of Modern Physics 54, no. 4 (1982): 1061

[20] Weinberg, S., The Quantum Theory of Fields., 1995 Cambridge

[21] J.Schwinger, "Sources and magnetic charge," Physical Review, Vol. 173, No.5, p.1536-1544, Sept.25, 1968

[22] H.J. Carmichael, Statistical Methods in Quantum Optics 2, Springer, New York, 2007.

[23] Huw Price, "Cosmology, time's arrow, and that old double standard." In Savitt, Steven F. (ed), Time's Arrow Today:Recent Physical and Philosophical Work on the Arrow of Time, Cambridge U. Press, 1997

[24] P. Werbos, Specification of the Q Hypothesis: An Alternative Mathematical Foundation for Physics. arXiv.org:quant-ph/0607096

[25] V.G.Makhankov, Yu.P.Rybakov and V.I.Sanyuk,The Skyrme Model, Springer-Verlag, 1994. 